\title{
Testing the Effectiveness of a Future Selves Intervention for Increasing Retirement Saving: Evidence from a Field Experiment in Mexico
}

Avni Shah ( $\sim$ Avni.Shah@rotman.utoronto.ca )

University of Toronto

Hal Hershfield

University of California Los Angeles

David Munguia Gomez

University of Chicago

Alissa Fishbane

idea42

Article

Keywords:

Posted Date: January 13th, 2022

DOI: https://doi.org/10.21203/rs.3.rs-1229969/v1

License: (1) (i) This work is licensed under a Creative Commons Attribution 4.0 International License. Read Full License 


\section{Abstract}

One psychological barrier impeding saving behavior is the inability to fully empathize with one's future self. Future self interventions have improved savings by helping people overcome this obstacle. Despite the promise of such interventions, previous research has focused predominantly on hypothetical contexts and western settings where the target sample has been predominantly undergraduate. Do interventions that encourage people to more concretely consider their future selves during retirement still have a positive effect on behavior in consequential, real-world savings decisions? Using a field experiment in Mexico $(N=7,603)$, where less than $1 \%$ make a voluntary savings contribution annually, we developed a low-cost, easy-to-implement intervention to test whether concrete thinking about one's future life improves recurring retirement savings signups relative to a status quo, control group. We find that future self decision aids significantly improved the likelihood of signing up for an automatic recurring savings plan by nearly four times compared to the control.

\section{Full Text}

Many parts of the world suffer from savings gaps, where citizens do not save at the rate needed to produces well-funded retirements. Psychological forces, such as an inability to fully emotionally connect with one's future self $(1,2)$, may be partially responsible for such gaps. One solution has come in the form of interventions meant to reduce the perceived interpersonal distance between one's present and future selves. Future self interventions - interventions where the salience of the future self is enhanced - have significantly improved savings intentions, as well as other long-term behaviors (3-5).

Though there has been much theoretical and practical interest in identifying the effectiveness of such interventions on retirement contribution decisions, the existing empirical evidence has focused predominately on laboratory settings with hypothetical outcomes and on cultural settings where saving for retirement is considered the norm. For example, one of the only field experiments to test the effectiveness of a future self intervention used a notably smaller sample $(\mathrm{N}=193)$ of employees at a private university in the United States (1). For many individuals, and in particular, for those outside of WEIRD (Westernized, Industrialized, Rich, and Democratic) cultures, retirement is considered a luxury, only afforded by a select few. Taken together, these observations raise important questions: Can future-self interventions significantly improve savings behavior at scale and across broader populations? And, do interventions that encourage people to elaborate on the future outcomes during retirement still have a positive effect, even in cultural settings where retirement may not be the norm?

It is imperative to determine the extent to which interventions can extend beyond laboratory settings and beyond WEIRD contexts, which represent just $12 \%$ of the world's population but over $90 \%$ of social psychology research samples $(6,7)$. Indeed, there has been a growing call for psychological theories to be tested in applied contexts in order to refine the theories and bolster practical applications that can inform pressing policy issues (e.g., 7-9). 
To address these questions, we partnered with a Mexican bank and employed a highly-powered field experiment in Mexico $(N=7,603)$-a country where less than $0.5 \%$ make a voluntary savings contribution annually (10). We investigated whether a future self intervention led participants to sign up for a recurring retirement savings program relative to the status quo policy, which simply asked individuals whether they would like to save for retirement.

To do so, we developed a tablet-based application comprising three key sections: a question exercise, a story, and a prompt to save more via setting up a direct debit (i.e., a recurring automatic withdrawal from a debit account into the retirement account). The interventions were delivered via the company's sales team, i.e., promoters, during meetings with account holders. In our primary intervention, individuals received questions that were meant to encourage concrete thinking about their future lives. In a second intervention, respondents were encouraged to think about their past as well as their futures, in an effort to remind them how previous actions may have benefited them in the present. The answers to these questions were then used in a "Madlibs"-style story about the account holder's life and aspirations for the future, piping in the account holder's responses to personalize the story (see Figure 1 and 2). We then measured whether this exercise influenced the likelihood that individuals set up a direct debit, automatically recurring savings deposit. Both future-self intervention conditions were compared to the status quo policy (i.e., the baseline condition). As indicated in our pre-registered hypotheses, we were agnostic as to whether the future-self intervention would be more or less effective than the past-self + future-self intervention, though we had assumed that both treatments would be more effective than the status quo.

Our main dependent variable of interest was the proportion who set up direct debit (i.e., a recurring voluntary retirement savings contribution). The study design, analysis plan, and hypotheses were preregistered (https://aspredicted.org/blind.php?x=xi2gy5).

\section{Results}

To investigate whether our interventions affected savings, we used a logistic regression analysis regressing the proportion who signed up for a recurring voluntary retirement savings contribution comparing each of the treatment conditions (Future and Future + Past) relative to the status quo, Control condition. The overall model was significant, $\chi^{2}(2,7603)=157.5, p<.001$. In comparison to the Control condition, both treatments significantly increased the proportion of account holders who made a voluntary retirement contribution. The Future + Past condition significantly increased the proportion making a voluntary retirement savings contribution (1.33\%), compared to the Contro/ condition $(0.11 \%), z$ $=6.49, p<.001$. And, the Future condition significantly increased the proportion signing up to make a voluntary retirement, and to a much higher degree relative to the Contro/ group, with the proportion signing up to make a voluntary contribution increasing from $0.11-4.45 \%, z=13.69, p<.001$.

Unexpectedly, promoters were slow to adopt either intervention, with over $80 \%$ of those in each of the intervention conditions occurring in the month of December. As a result, we went beyond our pre- 
registered analysis plan and conducted a multilevel logistic regression model to ensure that our results were robust to the inclusion of promoter-level and time-level (by month) controls. Table 2 presents multilevel regression model results with Models 1-3 capturing the regression output for our treatment conditions with our various controls. Across all models, both the Future and Future + Past conditions significantly increased the proportion signing up to make a voluntary retirement contribution relative to the Control group at the $p<.001$ level.

Our main analyses compared the likelihood of signing up for recurring savings for people who i) completed at least some of either intervention exercise, and ii) had received the intervention for the first time. We conducted further robustness checks using various sample exclusion criteria, including an intent-to-treat analysis in which we analyzed the full sample of individuals (including those who filled out none of the exercise as well as those who had multiple exposures to an intervention). These analyses are presented in detail in the Supplemental Materials. In all sample specifications, we find that both interventions significantly improved retirement contribution sign-ups at the $p<.01$ level.

In Table 3, we present the results of the multilevel regression models investigating the effectiveness of the two treatment conditions to one another, inclusive of control variables. Overall, the Future intervention significantly increased the proportion signing up to make a voluntary contribution, improving signups by over two times relative to the Future + Past treatment condition, with the proportion making a voluntary contribution increasing from $1.33-4.45 \%, \chi^{2}(1,2908)=26.46, p<.001$. We also perform several robustness checks ensuring that our results are robust to various sample exclusion criteria and time intervals which are presented in detail in the Supplemental Materials. Across all models, the Future condition significantly improves the proportion signing up to make a recurring voluntary savings contribution relative to the Future + Past treatment ( $p$ 's $<.001)$.

\section{Discussion}

Researchers across various disciplines such as psychology, philosophy, marketing, and economics have long argued that the degree to which individuals feel connected to their future selves can play a role in expectations for how much future utility they may feel in the present. Our work makes three main contributions in this space.

First, we provide some of the first field evidence demonstrating that improving the ability to imagine the future self significantly improves retirement savings behavior. Many interventions have drawn on these ideas but failed to scale up to the natural field environment, calling into question the strength or robustness of these effects. This is not just practically important: Examining whether theoretically-driven interventions are successful in field settings can, in itself, strengthen the theories from which they were developed (7-9). Given that naturalistic field experiments are considered to be the 'gold standard' of empirical evidence, our experiment provides the strongest test to date of whether increasing the salience of future selves can impact long-term retirement savings. 
Second, our results suggest that all intervention exercises that call to mind future selves may not be created equally. Despite the strong similarities in question prompts for the two treatment interventions, there was a significant increase in savings rates when people were given the future-self intervention alone compared to the past-self plus future-self one. Theoretically, in this past-self plus future-self condition, we used a more identifiable entity (the past self) to help people connect to a more difficult to imagine one (the future self, 1-5, 11). Even though this condition improved behavior relative to a traditional appeal, doing so was still less effective than having people solely elaborate on their future selves.

Why might this be the case? It is possible that elaborating on a more concrete past increased the focus of enjoying the present. Anecdotally, two participants mentioned previously being in bad relationships and then feeling liberated post-breakup. Elaborating on a past self that was worse off may have potentially increased the likelihood of exhibiting present bias, as people may have felt more licensed to indulge in the here and now. However, the field setting of this experiment made it difficult to determine the precise mechanism underlying the differential effectiveness of these two interventions. Future work should further explore why considering a future self alone may be more effective at changing behavior than thinking about a combination of past and future selves.

Finally, our results suggest that future self interventions are effective in novel cultural contexts. Mexico has historically low rates of retirement savings, with less than $1 \%$ of active account holders making a voluntary retirement contribution (9). Like in many other parts of the world, having the option of retiring from the workforce is a luxury only afforded or expected by a select few (12-13). Because many older individuals expect to be financially supported by their children or extended family, retirement saving is not typically prioritized (14-15). As a result, it is possible that our interventions could have been met with limited success in this context: encouraging people to elaborate on future selves who might ultimately be helped by younger generations could have just as likely lowered the motivation to save. And yet, our results suggest just the opposite. The effectiveness for future-self interventions are thus not only promising for those in Mexico but may also represent a potentially strong intervention for improving retirement savings behavior more broadly $(6,14-15)$.

From a policy perspective, it is important to note that the interventions we identified will not solve the lack of retirement savings alone. Increasing the propensity of making regular, small savings contributions nonetheless plays an important role in ensuring a more secure financial future. While other interventions require regulatory changes or more expensive additional forms of communication (e.g., SMS blasts or educational programs), the present one relies on a low-cost, easy-to-implement exercise (8). In particular, the exercise employed here did not require sophisticated technology or require participants to provide a photograph (e.g., 4, 11), making this intervention efficient from a time, labor, and financial standpoint.

An open question, however, concerns the extent to which such future-self interventions lead to lasting impact on behavior, or if they just provide a short-term boost in savings rates. Follow-up work investigating whether individuals continue with their recurring savings plan or not could be essential in understanding how to best motivate and sustain long-term behavioral change. 


\section{Methods}

\section{Background}

In response to significant reforms aimed at helping people save and prepare for their retirement, all fulltime workers in Mexico are required to select one of several government-designated retirement fund administrators known as AFOREs (Administradoras de Fondos para el Retiro) for their mandatory retirement contributions. We collaborated with one of the government-designated retirement companies for our field experiment. After selecting a company, workers were required to make monthly contributions to their retirement account, roughly equivalent to $6.5 \%$ of a worker's annual salary over the course of the year. Employers would also contribute a certain percentage on top of this mandatory amount. Historically, the combined total of the mandatory employee contributions, employer contributions, and the accrued interest earned would equate to receiving roughly $30 \%$ of the average earned salary at the time of retirement-far less than the $70 \%$ target that was typically recommended by financial experts (12-13). Account holders are encouraged help close the gap by making additional voluntary contributions to their retirement account, though less than $1 \%$ of account holders do so (10).

To help encourage voluntary savings contributions, most retirement companies hire salesforce teams, known as promoters, who serve as the main point of contact between the companies and the account holders (16-17). Promoters receive a commission for signing up new account holders and additional commission for persuading either existing or new account holders to sign-up for recurring voluntary savings contributions (16-17). Our experiment was conducted in collaboration with the retirement company, and was administered by promoters during their meetings with account holders from August 14th to December 22nd, 2017.

\section{Participants}

Participants were approached by promoters who were either assigned to the Future, Future + Past, or Control conditions. As indicated in our initial pre-registration plan, our main analyses were conducted with those account holders who i) completed at least some of the intervention exercise, and ii) had received the intervention for the first time. As a result, our main analysis excluded account holders who did not fill out any of the exercise and any accountholder responses beyond the first-time receiving the intervention. Our total sample, inclusive of those with incomplete and repeat responses, was 8,154 accountholders: 4,695 in the Control condition, 1,770 in the Future condition (Incomplete: $\mathrm{n}_{\text {Future }}=126$; Repeat participants: $\mathrm{n}_{\text {Future }}=162$ ), and 1,689 in the Future + Past condition (Incomplete: $\mathrm{n}_{\text {Future }}+$ Past $=182$; Repeat participants: $\mathrm{n}_{\text {Future }}+$ Past $=81$ ). Excluding the incomplete and repeat entries left us with a final sample of 7,603 individuals, with 4,695 in the Contro/ condition, 1,482 in the Future condition, and 1,426 in the Future + Past condition. It is worth noting that our two treatment interventions had significantly fewer participants due to initial failures in adopting the additional tablet app and technological difficulties navigating the app. To reduce any concerns of time-varying effects or bias driven due to this initial delay, 
we perform several robustness checks (included in the Supplemental Materials) and include month fixed effects to our models.

\section{Materials and Procedure}

Prior to starting the experiment, regardless of the condition to which they were eventually assigned, all promoters were required to attend a training session where they were informed that they would be part of a company-wide trial where their performance was being tracked. Promoters were told that they would be using their work-assigned tablet to go through exercises with their clients in order to help encourage them to save for retirement. Promoters were also informed that they might receive an additional tablet application with a follow-up exercise containing one of the intervention conditions that they also were instructed to administer to clients. Following the delivery of the exercises, promoters across all conditions were then instructed to ask whether clients wanted to set up for a recurring voluntary savings contribution via direct debit, all of which would also be recorded on the tablet device. If clients did choose to sign up for a recurring contribution, they (the clients) would indicate so on the tablet and would then be prompted to enter the relevant bank information and contribution amount accordingly.

Promoters were also told that their performance and sign-up rates were being tracked and monitored as per usual, regardless of the trial group to which they were assigned. All promoters were instructed and regularly reminded to promote signups of recurring voluntary retirement savings contributions via direct debit as part of their normal sales procedures.

Although all promoters were informed of the three trial conditions, each promoter was assigned to only one specific condition throughout the course of the experimental period (e.g., promoters assigned to the Future condition always delivered the Future version of the intervention). To avoid any selection effects, randomization occurred at the bank office level, meaning that each office had promoters in each of the three conditions. We also ensured that each of our three experimental conditions-Control, Future, and Future + Past-were roughly balanced, stratifying promoters within each bank into our three conditions based on seniority and productivity-level. In total, we randomized 345 promoters distributed across three regional offices (Mexico City metro area, Querétaro, and Monterrey). We stratified the promoter sample by region (two levels based on Central and Northern Mexico), seniority (three levels based on time at the company), and productivity (three levels based on the average account balance of previously acquired account holders). The random assignment yielded 117 promoters in the Contro/ condition, 110 in the Future condition, and 118 in the Future + Past condition. We found no differences in the stratification variables across conditions (see Table 1).

For the two future-self interventions, we designed and developed an additional tablet application that involved participants completing a question-and-answer exercise about how they envisioned their futures. Those in the Future condition only received questions about their future and future selves, while those in the Future + Past received questions about their pasts and past selves, as the same questions about the future from the Future condition. The answers to these questions were then piped into a MadLibs-style story that clients read aloud to their promoter. The precise text and question order for each condition 
(translated into English) as well as a screenshot of how the answers from the original Spanish exercise translated into a story are presented in Fig. 1 and Fig. 2, respectively. Given that Contro/ promoters did not have access to the intervention, they continued to use or developed their own individual approaches for signing up account holders for direct debit as per status quo.

Following the completion of the exercise, clients would then be taken to an additional screen where promoters would ask whether they wanted to set up a recurring voluntary savings contribution via direct debit. This screen was similar for all promoters, regardless of whether they were in the Control or either of the two intervention conditions.

\section{Data Availability}

Our experiments and analysis involve confidential financial data from Mexico, and thus they cannot be released publicly. We can arrange for individuals to work with the raw data for the purpose of replication on a secure computer after arranging for a non-disclosure agreement. The research team will facilitate this process for replication. We can also arrange for individuals to receive an anonymized dataset that deidentifies any individual information. The study design, analysis plan, and hypotheses for the field work were pre-registered and are all available at: https://aspredicted.org/blind.php?x=xi2gy5.

\section{References}

1. Bryan, C.J. \& Hershfield, H.E. You owe it to yourself: Boosting retirement saving with a responsibilitybased appeal. Decision 1 (S), 2 (2013)

2. Wilson, T.D. \& Gilbert, D.T. Affective forecasting: Knowing what to want. Current Directions in Psychological Science 14 (3), 131-134 (2005).

3. Bartels, D.M. \& Rips, L.J. Psychological connectedness and intertemporal choice. Journal of Experimental Psychology: General 139 (1), 49-69 (2010).

4. Hershfield, H.E., John, E.M. \& Reiff, J. S. Using vividness interventions to improve financial decision making. Policy Insights from the Behavioral and Brain Sciences 5 (2), 209-215.

5. Blouin-Hudon, E. M. C. \& Pychyl, T.A. Experiencing the temporally extended self: Initial support for the role of affective states, vivid mental imagery, and future self-continuity in the prediction of academic procrastination. Personality and Individual Differences 86, 50-56 (2015).

6. Henrich, J., Heine, S. J., \& Norenzayan, A. Most people are not WEIRD. Nature 466 (7302), 29 (2018).

7. Nielsen, M., Haun, D., Kärtner, J., \& Legare, C. H. The persistent sampling bias in developmental psychology: A call to action. Journal of Experimental Child Psychology 162, 31-38 (2017).

8. Walton, G.M. \& Wilson, T.D. Wise interventions: Psychological remedies for social and personal problems. Psychological Review 125 (5), 617 (2018).

9. Cohen, G. L., Garcia, J., \& Goyer, J. P. Turning point: Targeted, tailored and timely psychological intervention Ch. 35 in Elliot, A. J., Dweck, C. S., \& Yeager, D. S. (Eds.), Handbook of competence and 
motivation: Theory and application (Guilford Press, ed. 2, 2017).

10. Fertig, A., Lefkowitz, J. \& Fishbane, A. Using behavioral science to increase retirement savings. A New Look at Voluntary Pension Contributions in Mexico [Online]. Available at: URL http://www. ideas42. org/wp-content/uploads/2015/11/142_571_MexicoPensionsReport_ENG_final_digital. pdf [accessed November 21, 2020] (2015).

11. Hershfield, H.E., Goldstein, D.G., Sharpe, W.F., Fox, J., Yeykelis, L., Carstensen, L.L. \& Bailenson, J.N. Increasing saving behavior through age-progressed renderings of the future self. Journal of Marketing Research 48 (SPL), S23-S37 (2011).

12. Organisation for Cooperation and Development, OECD Reviews of Pension Systems: Mexico, OECD Reviews of Pension Systems. Organisation for Co-operation and Development, available at: https://doi.org/10.1787/9789264245938-en (2016).

13. Munnell, A.H. Golub-Sass, F. \& Webb, A. How much to save for a secure retirement. Center for Retirement Research at Boston College, available at: https://crr.bc.edu/wpcontent/uploads/2011/11/IB_11-13-508.pdf (2011).

14. Hofstede G. Culture's consequences: Comparing values, behaviors, institutions and organizations across nations (Sage Publications, 2001).

15. Triandis, H.C. Individualism and collectivism (Routledge, 2018).

16. Hastings, J.S., Hortaçsu, A. \& Syverson, C., Advertising and competition in privatized social security: The case of Mexico. NBER Working Paper Series, 18881 (2013).

17. Mitchell, O.S. Administrative costs in public and private retirement systems. In Feldstein, M. (Ed.), Privatizing Social Security 403-456 (University of Chicago Press, 1998).

\section{Tables}

Table 1. Promoter-Stratification Variables across Conditions

\begin{tabular}{|c|c|c|c|c|c|c|c|c|}
\hline \multirow[b]{2}{*}{ Condition } & \multicolumn{2}{|c|}{ Location } & \multicolumn{3}{|c|}{ Seniority } & \multicolumn{3}{|c|}{ Productivity } \\
\hline & Central & North & Low & Medium & High & Low & Medium & High \\
\hline Control & $37.61 \%$ & $62.39 \%$ & $14.29 \%$ & $42.86 \%$ & $42.86 \%$ & $13.27 \%$ & $41.84 \%$ & $44.90 \%$ \\
\hline Future & $38.52 \%$ & $61.48 \%$ & $14.29 \%$ & $40.95 \%$ & $44.76 \%$ & $16.19 \%$ & $40.00 \%$ & $43.81 \%$ \\
\hline \multirow[t]{2}{*}{ Future + Past } & $39.53 \%$ & $60.47 \%$ & $13.73 \%$ & $44.12 \%$ & $42.16 \%$ & $10.78 \%$ & $47.06 \%$ & $42.16 \%$ \\
\hline & \multicolumn{2}{|c|}{$\chi^{2}(2)=0.10, p=.953$} & \multicolumn{3}{|c|}{$\chi^{2}(4)=0.23, p=.994$} & \multicolumn{3}{|c|}{$\chi^{2}(4)=1.86, p=.762$} \\
\hline
\end{tabular}

*Stratification of these variables included only 305 of the 345 promoters. 
Table 2. Multi-level Logistic Regression, Proportion making a voluntary retirement savings contribution comparing the Future and Future + Past interventions versus the status quo, control condition across the inclusion of promoter-level and month-level control variables, Models 1-3.

\begin{tabular}{|c|c|c|c|c|c|c|}
\hline & \multicolumn{2}{|c|}{ Model 1} & \multicolumn{2}{|c|}{ Model 2} & \multicolumn{2}{|c|}{ Model 3} \\
\hline \multirow[t]{2}{*}{ Intercept } & -6.84 & $* * *$ & -6.84 & $* * *$ & -8.38 & $* * *$ \\
\hline & $(0.45)$ & & $(0.46)$ & & $(0.51)$ & \\
\hline \multicolumn{7}{|l|}{ Treatment Variable } \\
\hline \multirow[t]{2}{*}{ Future (vs. Control) } & 3.78 & $* * *$ & 3.78 & $* * *$ & 4.98 & $* * *$ \\
\hline & $(0.47)$ & & $(0.47)$ & & $(0.49)$ & \\
\hline \multirow[t]{2}{*}{ Future + Past (vs. Control) } & 2.54 & $* * *$ & 2.56 & $* * *$ & 3.67 & $* * *$ \\
\hline & $(0.50)$ & & $(0.50)$ & & $(0.52)$ & \\
\hline \multicolumn{7}{|l|}{ Controls } \\
\hline \multirow[t]{2}{*}{ Salesforce (Promoter) ID } & & & 0.00 & & 0.00 & \\
\hline & & & $(0.00)$ & & $(0.00)$ & \\
\hline \multirow[t]{2}{*}{ August } & & & & & 2.70 & $* * *$ \\
\hline & & & & & $(0.34)$ & \\
\hline \multirow[t]{2}{*}{ September } & & & & & 1.64 & $* * *$ \\
\hline & & & & & $(0.44)$ & \\
\hline \multirow[t]{2}{*}{ October } & & & & & 1.53 & $* * *$ \\
\hline & & & & & $(0.36)$ & \\
\hline \multirow[t]{2}{*}{ November } & & & & & 1.27 & $* * *$ \\
\hline & & & & & $(0.33)$ & \\
\hline $\mathrm{N}$ & 7603 & & 7548 & & 7548 & \\
\hline Chi-Square & 157.5 & $* * *$ & 156.5 & $* * *$ & 219.2 & $* * *$ \\
\hline
\end{tabular}

Note: Values represent $\beta$ coefficients with SE in parentheses. $*$ represents significance at the $p<.05$ level, $* *$ represents significance at the $p<.01$ level, and $* * *$ represents significance at the $p<.001$ level, respectively

Table 3. Multi-level Logistic Regression, Proportion making a voluntary retirement savings contribution comparing the Future vs. the Future + Past intervention across the inclusion of promoter-level, month-level, age, and gender controls, Models 1-5. 


\begin{tabular}{|c|c|c|c|c|c|c|c|c|c|c|}
\hline & \multicolumn{2}{|c|}{ Model 1} & \multicolumn{2}{|c|}{ Model 2} & \multicolumn{2}{|c|}{ Model 3} & \multicolumn{2}{|c|}{ Model 4} & \multicolumn{2}{|c|}{ Model 5} \\
\hline \multirow[t]{2}{*}{ Intercept } & -4.31 & $* * *$ & -4.35 & $* * *$ & -4.77 & $* * *$ & -4.41 & $* * *$ & -4.56 & $* * *$ \\
\hline & $(0.23)$ & & $(0.27)$ & & $(0.29)$ & & $(0.52)$ & & $(0.54)$ & \\
\hline
\end{tabular}

\section{Treatment Variable}

Future (vs. Future + Past)

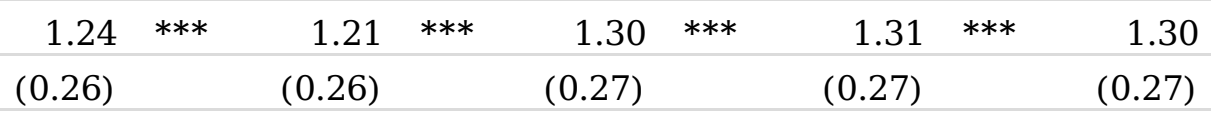

\section{Controls}

Salesforce (Promoter) ID

$\begin{array}{rrrr}0.00 & 0.00 & 0.00 & 0.00 \\ (0.00) & (0.00) & (0.00) & (0.00)\end{array}$

Month = August

2.65

$* * * \quad 2.62$

$* * *$

2.59

Month $=$ September

(0.36)

(0.36)

(0.36)

$1.16 *$

(0.55)

$*$

$1.14 *$

$1.16 *$

Month = October

1.61

(0.55)

(0.55)

Month = October

(0.36)

1.57

1.58

Month $=$ November

1.34

(0.36)

(0.36)

(0.33)

1.34

1.35

(0.33)

(0.33)

Age

$-0.01$

$-0.01$

(0.01)

(0.01)

Female

0.32

(0.23)

$\mathrm{N}$

2908

2853

2853

2853

2853

Chi-Square

26.46

$25.68 * * *$

$84.24 \quad * * *$

$84.92 \quad * * * \quad 86.84$

Note: Values represent $\beta$ coefficients with SE in parentheses. * represents significance at the $p<.05$ level, $* *$ represents significance at the $p<.01$ level, and $* * *$ represents significance at the $p<.001$ level, respectively.

\section{Figures}

\section{Figure 1}

Question Order for Treatment Interventions, Future and Future + Past (English translation)

Figure 2 
Screenshot of Question and Story from the Future-self Intervention. Note. Sample screens from the Future only condition. Panel A (left) shows the first eight questions that the promoter asked the account holder. Panel $B$ (right) shows an excerpt of the story that the account holder read out loud.

\section{Supplementary Files}

This is a list of supplementary files associated with this preprint. Click to download.

- 01042022TestingEffectivenessFutureSupplementNHB.docx 